\title{
FIXED POINT THEOREMS FOR ASYMPTOTICALLY PSEUDOCONTRACTIVE MAPS IN CERTAIN BANACH SPACES
}

DONATUS I. IGBOKWE

(Received 28 February, 2003; Revision accepted 13 April. 2004)

\section{ABSTRACT}

Let $E$ be a real $q$-uniformly smooth Banach space (for example, $L_{p}$ or $1 p$ spaces, $1<p<\infty$ ) and let $T: E \rightarrow E$ be asymptotically pseudocontractive mapping with nonempty fixed point set $F(T)$ and a real sequence $\left\{k_{n}\right\}_{n=1}^{\infty}$. Let $\left\{x_{n}\right\}_{n=1}$ be the sequence generated from an arbitrary $x_{1}$ in $E$ by $x_{n+1}=\left(1-\alpha_{n}\right) x_{n}+\alpha_{n} T^{n} x_{n}, n \geq 1$. If the range of $T$ is bounded and there exists a strictly increasing funciion $\phi:[0, \infty) \rightarrow[0, \infty)$ with $\Phi(0)=0$ such that $\left\langle T^{n} x_{n}-p, j\left(x_{n}-p\right)\right\rangle \leq k_{n}\left\|x_{n}-p\right\|^{2}-\Phi\left(\| x_{n}-\right.$ $p \mid l), p \in F(T)$, then $\left\{x_{n}\right\}_{n=1}^{\infty}$ converges strongly to $p$, provided that $\left\{k_{n}\right\}$ and $\left\{\alpha_{n}\right\}$ satisfy certain properties. This result compliments the results of Chang, Park and Cho (2000), by dropping the Lipschitz condition on $T$.

\section{Mathematics Subject Classification : 47H09 47J05.}

KEY WORDS: Fixed points, asymptotically pseudocontractive maps, modified Mann iterative methods,

$$
\text { q-uniformly smooth spaces. }
$$

\section{INTRODUCTION}

Let $E$ be an arbitrary real Banach space and let $J_{q}(q>1)$ denote the generalized duality mapping from $E$ into $2^{E^{*}}$ given by $J_{q}(x)=\left\{f \in E^{*}:\langle x, f\rangle=\|x\|^{q}\right.$ and $\left.\|f\|=\|x\|^{q-1}\right\}$, where $E^{*}$ denotes the dual space of $E$ and $\langle.,$.$\rangle denotes the genaralised duality pairing. In particular, \mathrm{J}_{2}$ is called the normalized duality mapping and is usually denoted by J. It is well known (see for example Xu (1991)) that $d_{q}(x)=\|x\|_{q-2} J(x)$ if $x \neq 0$, and that if $E$ is uniformly smooth then $J q$ is single-valued and uniformly continuous on bounded sets. In the sequel we shall denote the single-valued generalized duality mapping by $\mathrm{j}_{\mathrm{q}}$.

Let $E$ be a real Banach space. The modulus of smoothness of $E$ is the function $\rho E:[0, \infty) \rightarrow[0, \infty)$ defined by

$$
\rho t(\tau)=\sup \{1 / 2(\|x+y\|+\|x-y\|)=1:\|x\| \leq 1,\|y\| \leq \tau\} \text {. }
$$

$E$ is uniformy smooth if and only if $\lim \left(\rho_{E}(\tau) / \tau\right)=0$ as $\tau \rightarrow 0$. Hilbert spaces, $L F$ (or $(p)$ spaces, with $1<p<\infty$, and the Sobolev spaces, $W_{m}^{p} m$ a positive integer, and $1<p<\infty$, are $q$. uniformly smooth. Hiberi speces are 2-uniformly smooth while

$$
L p(\text { or } l p) \text { or } W_{m}^{p} \text { is }\left\{\begin{array}{l}
p \text {-uniformly smooth if } 1<p \leq 2 \\
2-1 \text { iniformly smooth if } p \geq 2 .
\end{array}\right.
$$

The following theorem gives a geometric characterization of q-uniformly smooth Banach spaces : 
Theorem HKX (Xu (1991), P.1130): Let $q>1$ and let $E$ be a real Banach space. Then the following statements are equivalent:

1. E is q-uniformly smooth

2. There exists a constant $c_{q}>0$ such that for all $x, y \in E$

$$
\|x+y\|^{q} \leq\|x\|^{q}+q<y, j_{q}(x)>+c_{q}\|y\|^{q}
$$

3. There exists a constant $d_{q}$ such that for all $x, y \in E$, and $t \in[0,1]$

$\left.\|(1-t) x+t y\|^{q} \geq(1-t)\|x\|^{q}+t\right] \mid y\left\|^{q}-w_{q}(t) d_{q}\right\| x-y \|^{q}$,

where $w_{q}(t)=t^{9}(1-t)+t(1-t)^{q}$.

Let $K$ be a nonempty subset of an arbitrary real Banach space $E$. A mapping $T: K \rightarrow K$ is called asymptotically pseudocontractive with sequence $\left\{k_{n}\right\}$ $\subseteq[1, \infty), \lim _{n \rightarrow \infty} k_{n}=1$ (see Schu (1991), Chang et al (2000), Osilike and Igbokwe (2002)) if for all $x, y \in K$, there exists $j(x-y) \in J(x-y)$ such that

$$
\left\langle T^{n} x-T^{n} y, j(x-y)\right\rangle \leq k_{n}\|x-y\|^{2}
$$

for all $\mathrm{n} \in \mathrm{N}$. As shown in Schu (1991), this class of mapping is more general than the important class of asymptotically nonexpansive mapping (ie mappings $T: K \rightarrow K$ such that $\left\|T^{n} x-T^{n} y\right\| \leq k_{n} \| x-$ $y \|$, for all $x, y \in K$, for some sequence $\left\{K_{n}\right\}$ with $\lim k_{n}=1, n \rightarrow \infty$ and for all $n \in N$ ) introduced by Goebel and Kirk (1972). T is called uniformly L-Lipschitzian if $\left\|T^{n} x-T^{n} y\right\| \leq L\|x-y\|$, for all $x, y \in$ $K, n \in N$ and for some $L>0$.

The class of asymptotically pseudocontractive maps was introduced by Schu (1991). In (1991) Schu also introduced the modified Mann iteration method $\left\{x_{n}\right\}$ generated from an arbitrary $x_{1} \in K$ by

$$
x_{n+1}=\left(1-\alpha_{n}\right) x_{n}+\alpha_{n} T^{n} x_{n}, n \geq 1,
$$

where $\left\{\alpha_{n}\right\}$ is a real sequence in $[0,1]$.

In 2000, Chang, Park and Cho proved the following theorem:

Theorem CPC (Chang et al (2000), Theorem 2.3): Let $K$ be a nonempty closed convex subset of $E$. Let $T: K \rightarrow K$ be a uniformly $L$-Lipschitzian asymptotically pseudocontractive mapping with a sequence $\left\{k_{n}\right\}$ in $[1, \infty)$ such that $\lim k_{n}=1$. 
Let $\left\{a_{n}\right\}$ be a sequence in $[0,1]$ satisfying the following conditions:

(i) $\alpha_{n} \rightarrow 0$ as $n \rightarrow \infty$

(ii) $\quad \sum_{n=1}^{\infty} \alpha_{n}=\infty$.

Let $\left\{x_{n}\right\}$ be the modified Mann iterative sequence defined by (4). If $F(T)=\{x \in K: T x=x\} \neq$ $\phi$, and if for any given $p \in F(T)$, there exists a strictly increasing function $\phi:[0, \infty) \rightarrow[0, \infty)$ with $\phi(0)=0$ such that

$$
\left\langle T^{n} x_{n+1}-p, j\left(x_{n+1}-p\right)\right\rangle \leq k_{n}\left\|x_{n+1}-p\right\|^{2}-\phi\left(\left\|x_{n+1}-p\right\|\right)
$$

for all $n \geq 1$, where $j\left(x_{n+1}-p\right) \in J\left(x_{n+1}-p\right)$ is such that $\left\langle T^{n} x_{n+1}-T^{n} p, j\left(x_{n+1}-p\right)\right\rangle \leq k_{n}\left\|x_{n+1}-p\right\|^{2}$, $\forall n \geq 1$, then the sequence $\left\{x_{n}\right\}$ converges strongly to the fixed point $p$ of $T$.

The assumption that $T$ is uniformly $L$-Lipschtizian appears restrictive since it is not satisfied by many continuous maps.

It is our purpose in this paper to enlarge the class of operators satisfying Theorem CPC by dropping the uniform L-Lipschitizian condition. No continuity assumption will be made on the operator T. Furthermore, we shall extend the result to the more general modified Mann iteration methods with errors in the senses of Liu (1995) and Xu (1998). We shall prove our result in q-uniformly smooth spaces. Our method of proof is short. Our results complement those of Chang, Park and Cho (2000) and a very recent result of Osilike and Igbokwe (2002).

\section{MAIN RESULTS}

In the sequel, we shall require the following Lemma:

Lomma 2.1: Let $\left\{a_{n}\right\},\left\{b_{n}\right\}$ and $\left\{t_{n}\right\}$ be nonnegative real sequences such that $\Sigma t_{n}=\infty, t_{n} \rightarrow 0$ as $n \rightarrow \infty, b_{n}=0\left(t_{n}\right)$ and satisfy

$$
a_{n+1}^{q} \leq a_{n}^{q}-t_{n} \phi\left(a_{n}\right)+b_{n} n \geq 1
$$

where $\phi:[0, \infty) \rightarrow[0, \infty)$ is a strictly increasing function with $\phi(0)=0$. Then $a_{n} \rightarrow 0$ as $n \rightarrow \infty$. Proof: The prove of the lemma follows from the following two claims:

Claim 1: $\lim \inf a_{n}=0$.

Assume the contrary and let $\lim$ inf $a_{n}=\delta>0$. Then there exists a positive integer $N_{0}$ such that $\forall n \geq N_{0}, a_{n}>\delta / 2$. Also $b_{n}=O\left(t_{n}\right)$ implies that $\exists N_{1}>0$ such that $b_{n} / t_{n} \leq 1 / 2$ $\phi(\delta / 2)\left(i \Theta b_{n} \leq 1 / 2 \phi(\delta / 2) t_{n}\right), \forall n \geq 1$. Define $N \max \left\{N_{0}, N_{1}\right\}$. Then $\forall n \geq N,(6)$ implies

$$
\begin{aligned}
a_{n+1}^{q} & \leq a_{n}^{q}-t_{n} \phi(\delta / 2)+1 / 2 \phi(\delta / 2) t_{n} \\
& \leq a_{n}^{q}-1 / 2 \phi(\delta / 2) t_{n} .
\end{aligned}
$$

so that

$$
1 / 2 \phi(\delta / 2) t_{n} \leq a_{n}^{q}-a_{n+1}^{q},
$$


Therefore, $\forall \mathbf{n} \geq \mathbf{N}$,

$$
1 / 2 \phi(\delta / 2) \sum_{j=N}^{n} t_{j} \leq a^{q} n \text {. }
$$

Hence, $\Sigma t_{n}<\infty$, contradicting $\Sigma t_{n}=\infty$. Thus lim inf $a_{n} \approx 0$. Therefore, there exists a subseruence $\left\{a_{n j}\right\}$ of $\left\{a_{n}\right\}$ such that $a_{n j} \rightarrow 0$ as $j \rightarrow \infty$. Thus given any $\varepsilon>0$ there exists an integer jo $>0$ such that $\forall j \geq j 0$ jij $a_{n j}<\varepsilon / 2$. Also, $\exists N_{2}>0$ such that $\forall n \geq N_{2}, b_{n}<1 / 2 \phi(\varepsilon / 2) t_{n}$.

Denote $n_{j *}$ by $n^{*}$ and set $n^{*} \geq \max \left\{n_{j *}, N_{2}\right\}$.

Claim 2: $\quad a_{n+k}<\varepsilon / 2, \forall k \geq 0$.

For $k=0$, the claim is clearly true. We assume that the claim is true for $30 m e k>0$. Suppose the claim is false for $k+1$. Then $a_{n+k+1} \geq \varepsilon / 2$. But from (6)

$$
\begin{aligned}
\varepsilon^{q} / 2^{q} \leq a^{q} n^{*+k+1} & \leq a^{q} n^{*+k}-t_{n^{*}+k} \phi\left(a_{n^{*}+k+1}\right)+b_{n^{*}+k} \\
& <(\varepsilon / 2)^{q}-t_{n^{*}+k} \phi(\varepsilon / 2)+1 / 2 t_{n^{*}+k} \phi(\varepsilon / 2) \\
& =\varepsilon^{q} / 2^{q}-1 / 2 \phi(\varepsilon / 2) t_{n^{*}+k}<\varepsilon^{q} / 2^{q},
\end{aligned}
$$

which is a contradiction. Claim 2 is therefore proved. Since $\varepsilon>0$ is arbitrary, this implies that an $\rightarrow 0$ as $n \rightarrow \infty$, completing the proof of the lemma.

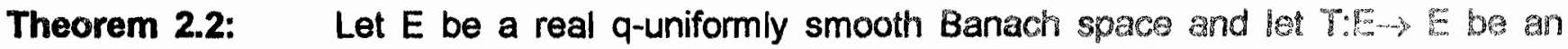
asymptotically pseudocontractive mapping with a nonempty fixed point set $\mathrm{F}(\mathrm{D})$ and a real sequence $\left\{k_{n}\right\}$ in $[1, \infty)$ such that $\lim k_{n}=1, n \rightarrow \infty$. Let $\left\{\alpha_{n}\right\}$ be a real sequence in $[0,1]$ satisfying (i) $\lim \alpha_{n}=0, n \rightarrow \infty$ and (ii) $\Sigma \alpha_{n}=\infty$. Let $\left\{x_{n}\right\}$ be the sequence generated from an arbitrary $x_{4}$ E E by

$$
x_{n+1}=\left(1-\alpha_{n}\right) x_{n}+\alpha_{n} T^{n} x_{n}, n \geq 1
$$

If the range of $T$ is bounded and if for some $p \in F(T)$ there exists a stricily increasing function $\phi:[0, \infty) \rightarrow[0, \infty)$ with $\phi(0)=0$ such that

$$
\left\langle T^{n} x_{n}-p, j\left(x_{n}-p\right)\right\rangle \leq k_{n}\left\|x_{n}-p\right\|^{2}-\phi\left(\left\|x_{n}-p\right\|\right) \text {, }
$$

then $\left\{x_{n}\right\}$ converges strongly to $p$.

Proof: $\quad$ Set $M \doteq \operatorname{isup}_{n \geq 1}\left\|T^{n} x_{n}-p\right\|$. Using (4), (1) and (5) we obtain:

$$
\begin{aligned}
\left\|x_{n+1}-p\right\|^{q}= & \|\left(i-\left(x_{n}-p\right)+\alpha_{n}\left(T^{n} x_{n}-p\right) \|^{q}\right. \\
\leq & \left(1-\alpha_{n}\right)^{q}\left\|x_{n}-p\right\|^{q}+q\left(1-\alpha_{n}\right) \alpha_{n}\left(T^{n} x_{n}-p, \operatorname{In}\left(x_{n}-p\right)\right\rangle \\
& +c_{q} \alpha_{n}^{q}\left\|T^{n} x_{n}-p\right\|^{q} \\
\leq & {\left[\left(1-\alpha_{n}\right)^{q}+q\left(1-\alpha_{n}\right) \alpha_{n} k_{n}\right]\left\|x_{n}-p\right\|^{q} } \\
& -q\left(1-\alpha_{n}\right) \alpha_{n} \phi\left(\left\|x_{n}-p\right\|\right)+c_{q} \alpha_{n}^{q} M^{q}
\end{aligned}
$$




$$
\begin{aligned}
\leq & {\left[1-q \alpha_{n}+\alpha_{n}^{q}+q\left(1-\alpha_{n}\right) \alpha_{n} k_{n}\right]\left\|x_{n}-p\right\| } \\
& -q\left(1-\alpha_{n}\right) \alpha_{n} \phi\left(\left\|x_{n}-p\right\|\right)+c_{q} \alpha_{n}^{q} M^{q} \\
\leq & {\left[1+q \alpha_{n}\left(x_{n}-1\right)\right]\left\|x_{n}-p\right\|^{q}-q\left(1-\alpha_{n}\right) \alpha_{n} \phi\left(\left\|x_{n}-p\right\|\right) } \\
& +c_{q} \alpha_{n}^{q} M^{q}
\end{aligned}
$$

Let $D \doteq \sup \left\{\left\|T^{n} x_{n}-p\right\|, n \geq 1\right\}+\left\|x_{1}-p\right\|$. Then by simple induction $\left\|x_{n}-p\right\| \leq D, \forall n$ $\geq 1$.

It follows from (9) that

$$
\left\|x_{n+1}-p\right\|^{q} \leq\left\|x_{n}-p\right\|^{q}-q\left(1-\alpha_{n}\right) \alpha_{n} \phi\left(\left\|x_{n}-p\right\|\right)+b_{n,} \quad \forall n \geq 1
$$

where

$$
b_{n}=c_{q} \alpha_{n}^{q} M^{q}+q \alpha_{n}\left(k_{n}-1\right) D^{q}
$$

Now, if we set $a_{n} \doteq\left\|x_{n}-p\right\|$, and $t_{n} \doteq q\left(1-\alpha_{n}\right) \alpha_{n}$, in (10) we obtain

$$
a_{n+1}^{q} \leq a_{n}^{q}-t_{n} \phi\left(a_{n}\right)+b_{n}, \quad \text { which is (6). }
$$

Thus by lemma $2.1, x_{n} \rightarrow p$ as $n \rightarrow \infty$, completing the proof of theorem 2.2 .

Remark : Once convergence results have been proved for the original modified Mann iteration method (4), the extensions of the results to the modified iteration method with errors in the sense of Liu (1995) and Xu (1998) are usually straight forward on imposing the necessary conditions on the error term. Hence, we have the following results whose proofs are omitted because they follow by straight forward modifications of the proofs on the corresponding results for the original modified Mann iteration method (4).

Theorem 2.3: Let $E, T$ and $\alpha_{n}$ be as in Theorem 2.2. Let $\left\{x_{n}\right\}$ be the sequence generated from an arbitrary $x_{1} \in E$ by

$$
x_{n+1}=\left(1-\alpha_{n}\right) x_{n}+\alpha_{n} T^{n} x_{n}+u_{n}, \quad n \geq 1
$$

where $\Sigma\left\|u_{n}\right\|<\infty$. If the range of $T$ is bounded and if for some $p \in F(T)$, there exists a strictly increasing function $\phi:[0, \infty) \rightarrow[0, \infty)$ with $\phi(0)=0$ such that

$$
\left\langle T^{n} x_{n}-p, j\left(x_{n}-p\right)\right\rangle \leq k_{n}\left\|x_{n}-p\right\|^{2}-\phi\left(\left\|x_{n}-p\right\|\right)_{i}
$$

then $\left\{x_{n}\right\}$ converges strongly to $p$.

Theorem 2.4: Let $E$ and $T$ be as in theorem 2.2. Let $\left\{x_{n}\right\}$ be the sequence generated from an arbitrary $x_{1} \in E$ by

$$
x_{n+1}=a_{n} x_{n}+b_{n} T^{n} x_{n}+c_{n} u_{n} \quad n \geq 1 \text {. }
$$

where (i) $a_{n}+b_{n}+c_{n}=1$ (ii) $\Sigma b_{n}=\infty, \lim b_{n}=0$ and (iii) $\Sigma c_{n}<\infty$

If $\left\{u_{n}\right\}$ is a bounded sequence in $E$, the range of $T$ is bounded and for wome $p e$ for there exists a strictly increasing function $\phi:[0, \infty) \rightarrow[0, \infty)$ with $\phi(0)=0$ such that 
$\left\langle T^{n} x_{n}-p, j\left(x_{n}-p\right)\right\rangle \leq k_{n}\left\|x_{n}-p\right\|^{2}-\phi\left(\left\|x_{n}-p\right\|\right)$.

then $\left\{x_{n}\right\}$ converges strongly to $p$.

Set $\alpha_{n}=b_{n}+c_{n}$ in (11) to obtain

$$
x_{n+1}=\left(1-\alpha_{n}\right) x_{n}+\alpha_{n} T^{n} x_{n}+c_{n}\left(u_{n}-T^{n} x_{n}\right), \quad \forall n \geq 1 .
$$

The rest of the proof follows as in the proof of Theorem 2.2 .

\section{REFERENCES}

Chang, S. S., Park J. Y. and Cho, Y. J., 2000. Iterative approximations of fixed points of asymptoticaily nonexpansive mappings in Banach spaces, Bulletin of Korean Mathematical Society, $3 \uparrow(1): 109-119$.

Goebel K. and Kirk W. A., 1972. A fixed point theorem for asymptotically nonexpansive mappings, Proceedings of American Mathematical Society, 35: 171-174.

Liu, L. S., 1995. Ishikawa and Mann iterative processes with errors for nonlinear strongly accretive mappings in Banach spaces, Journal of Mathematical Analysis and Application, 194: 114 - 125.

Osiike, M. O. and Igbokwe, D. I., 2002. Convergence theorems for asymptotically pseudocontractive maps, Bulletin of Korean Mathematical Society, 32(3): 389- 399.

Schu, J., 1991. Iterative construction of fixed points of asymptotically non expansive mappings, Journal of Mathematical Analysis and Application, 158: 409-413.

Xu, H. K., 1991. Inequalities in Banach spaces with applications, Nonlinear Analysis. 16: 1127 - 1138.

$\mathrm{Xu}, \mathrm{Y}$, 1998. Ishikawa and Mann iterative methods with errors for nonlinear operator equation, Journal of Mathematical Analysis and Application, 224: $91-101$. 